\title{
Hematological Changes in Sudanese Patients with Falciparum Malaria Attending Elnihoud Teaching Hospital
}

Corresponding Author: Hafiz Ahmed Hobiel; email: hebiel78@yahoo.com

Received 15 January 2019 Accepted 26 March 2019 Published 31 March 2019

Production and Hosting by Knowledge $B$

(c) Abdelnassir M. Ahamed et al. This article is distributed under the terms of the Creative Commons

Attribution License, which permits unrestricted use and redistribution provided that the original author and source are credited.

Editor-in-Chief:

Prof. Mohammad A. M. Ibnouf
Abdelnassir M. Ahamed ${ }^{1}$, Hafiz Ahmed Hobiel ${ }^{2}$, Gad Allah Modawe ${ }^{3}$, and Mohammed Saeed Elsammani ${ }^{4}$

${ }^{1}$ Department of Pathology, Faculty of Medicine and Health Sciences, University of West Kordufan, Elnihoud, Sudan

${ }^{2}$ Department of Biochemistry, Faculty of Medicine and Health Sciences, University of West Kordufan, Elnihoud, Sudan

${ }^{3}$ Department of Biochemistry, Faculty of Medicine and Health Sciences, Omdurman Islamic University, Omdurman, Sudan

${ }^{4}$ Department of Pathology, Faculty of Medicine, University of Albaha, Saudi Arabia

\section{Abstract}

Backgrounds: Malaria is a major public health problem in the tropical and subtropical areas of the world, including Africa. Most cases of malaria in Africa are caused by Plasmodium falciparum.

Objectives: This study was aimed to assess the hematological changes in patient with falciparum malaria and to estimate the incidence of leucopenia, thrombocytopenia, and its response to anti-malaria therapy, and to correlate the association of the hematological changes with $P$. falciparum-positive (study group) and $P$. falciparumnegative (control group).

Methodology: A total of 453 participants (353 cases and 100 as control group) were enrolled in this study, all of them were randomly selected from Elnihoud Teaching Hospital, Elnihoud Locality, West Kordufan State, Sudan. Questionnaire was filled by every participant and thick and thin blood films for malaria were prepared and stained by giemsa stain and the CBC was done by sysmex automated hematological analyzer. Results: Thrombocytopenia, leucopenia, microcytic hypochromic red blood cells and relative lymphocytosis have significant association with falciparum malaria with $P$ values $0.008,0.001,0.008$, and 0.004 , respectively.

Conclusion: Patients with thrombocytopenia and/or leucopenia with malaria should receive anti-malaria therapy and follow-up by $\mathrm{CBC}$, and shouldn't be hurried for a bone marrow examination.

Keywords: falciparum malaria, thrombocytopenia, leucopenia, relative lymphocytosis

\section{G OPEN ACCESS}




\section{Introduction}

Malaria is a major public health problem in the tropical and subtropical parts of the world [1]. It is one of the most common dangerous protozoan disease in which most cases in Africa are caused by $P$. falciparum, with a relatively small proportion attributable to $P$. malariae or $P$. ovale, while $P$. vivax is known to occur in only some areas of the region [2]. An estimated 219 million cases of malaria occurred worldwide in 2017 compared with 239 million cases in 2010 and 217 million cases in 2016. The WHO African region still bears the largest burden of malaria morbidity, with 200 million cases (92\%) in 2017, followed by the WHO South-East Asia Region (5\%) and the WHO Eastern Mediterranean Region (2\%) [3].

The clinical manifestation of falciparum malaria varies from mild acute febrile illness to life-threatening severe systemic complications involving one or more organ systems [4]. It causes significant hematological changes ranging from hemolytic anemia $(\mathrm{Hb}<$ $10 \mathrm{~g} / \mathrm{dl}$.), defective erythropoiesis, and reticulocyte production, leukocytosis (WBC > 11 X 103/Microliter), leukocytopenia (WBC $<4$ X103/Microliter), thrombocytopenia (TCP) platelets count less than $150 \times 103 /$ Microliter, platelet dysfunction in severe malaria, and disseminated intravascular coagulation (DIC) (5). Severe TCP is frequently noticed with P. falciparum Malaria [5]. TCP during malarial infection may appear even before fever, anemia, and splenomegaly become manifest [6]. During early stages of malaria, platelet agglutination as a result of endothelial cell activation and release of activated von Willebrand factor occur which may cause thrombocytopenia [7]. Direct invasion of platelets by malarial parasites may occur. Thrombocytopenia in malaria is usually mild and treated by eradication of malaria parasite [8]. Thrombocytopenia is an early and consistent feature of malaria, but its pathogenesis remains incompletely understood [9]. The causes of thrombocytopenia in falciparum malaria are increased platelet consumption as evidenced by shortened survival of platelets and the finding of increased megakaryocytes in patient's bone marrow and elevated plasma thrombopoietin levels [10], and systemic microvascular sequestration and endothelial activation may play a pathophysiological role, a hypothesis supported by the observation that the radiolabelled platelets of patients with falciparum malaria are diffusely sequestered rather than pooling in the liver or spleen [11]. Population studies have shown an association between thrombocytopenia and outcome, and a recent study from India proposed that thrombocytopenia should be added to the World Health Organization (WHO) criteria for the definition of severe malaria [12]. This study was aimed to assess the hematological changes in patients with falciparum malaria. 


\section{Materials and Methods}

This study was an analytical cross-sectional study, carried out in Elnihoud Teaching Hospital, Elnihoud Locality, West Kordufan State, Sudan, from April 2015 to April 2016. Elnihoud Teaching Hospital is tertiary referring hospital receiving approximately about 15,000 patients suffering from malaria annually. A total of 453 participants (353 cases and 100 as control group) were enrolled in this study, all of them were randomly selected. Participants with positive blood film for malaria were taken as cases group, and febrile patients with a negative result for malaria were taken as control group, any patient with hematological disorders was excluded. The institutional research and ethics approval was obtained before the commencement of the study. All participants spoke sufficient Arabic to provide informed consent. Questionnaires were filled, blood samples were obtained for the detection of malaria using giemsa-stained thick- and thin blood films and stander diagnostic (SD) ICT for malaria antibodies. Full blood count was done for the patients with abnormal blood count two weeks after completing the anti-malaria therapy; the complete blood count ( $\mathrm{CBC}$ ) was analyzed by automated hematological analyzer (sysmex XP-300); also the peripheral blood film was stained by Leishman's stain and screened by an expert hematologist. The data were analyzed by SPSS program version 20.

\section{Results}

The distribution of the ages of the study participants were between 4 months and 75 years with the mean of $29+/-3.4$ years. Of the total participants, 216 (88.5\%) were rural while 28 (11.5\%) were urban. The study results show that the incidence of pancytopenia among study group was 6 out of 353 (1.7\%) (Table 1).

The incidences of anemia, leucopenia, and thrombocytopenia among the study group were: 77 out of 353 (21.8\%), 22 out of 352 (6.3\%), and 75 out of 353 (21.3\%), respectively. Whereas, on the other hand, the incidences of microcytic hypochromic red blood cells and relative lymphocytosis (the absolute lymphocyte count is normal but it is more than neutrophil count) among study group were 140 out of 353 (39.7\%) and 51 out of 267 (19.1\%), respectively. There was a statistically significant positive association between $P$. falciparum malaria and leucopenia, relative lymphocytosis, thrombocytopenia and microcytic hypochromic red blood cells with $P$-values $0.001,0.004,0.008$, and 0.008 , respectively (Table 2). Two weeks' follow-up after completing the treatment, 90.7\% of thrombocytopenic and $90.9 \%$ of thrombocytosis patients showed normal platelet count. 
For WBCs, $90.9 \%$ of leukopenia and $86.5 \%$ of leukocytosis patients revealed normal value (Table 3).

TABLE 1: Characteristics and description of the study groups.

\begin{tabular}{l} 
Characteristic \\
Age \\
Under 5 years \\
5 to 14 years \\
15 to 50 years \\
Above 50 years \\
Total \\
Sex \\
Male \\
Female \\
Total \\
Residence Area \\
Rural \\
Urban \\
Total \\
Incidence of \\
pancytopenia \\
Present \\
Absent \\
Total \\
\hline
\end{tabular}

\begin{tabular}{|c|}
\hline Patient \\
\hline $37(12 \%)$ \\
\hline $46(14.9 \%)$ \\
\hline $164(53.1 \%)$ \\
\hline $62(20.1 \%)$ \\
\hline $309(100 \%)$ \\
\hline $188(76.7 \%)$ \\
\hline $165(79.7 \%)$ \\
\hline $353(100 \%)$ \\
\hline $216(88.5 \%)$ \\
\hline $28(11.5 \%)$ \\
\hline $244(100 \%)$ \\
\hline $6(1.7 \%)$ \\
\hline $347(98.3 \%)$ \\
\hline $353(100 \%)$ \\
\hline
\end{tabular}

\begin{tabular}{|l|}
\hline Control \\
\hline $33(24.7 \%)$ \\
\hline $12(12.6 \%)$ \\
\hline $34(35.8 \%)$ \\
\hline $16(16.8 \%)$ \\
\hline $95(100 \%)$ \\
\hline $57(23.3 \%)$ \\
\hline $42(20.3 \%)$ \\
\hline $99(100 \%)$ \\
\hline $68(81.9 \%)$ \\
\hline $15(18.1 \%)$ \\
\hline $83(100 \%)$ \\
\hline $0(0.00 \%)$ \\
\hline $100(100 \%)$ \\
\hline $100(100 \%)$ \\
\hline
\end{tabular}

\begin{tabular}{|c|}
\hline Total \\
\hline $70(17.3 \%)$ \\
\hline $58(14.4 \%)$ \\
$198(49 \%)$ \\
\hline $78(19.3 \%)$ \\
\hline $404(100 \%)$ \\
\hline $245(54.2 \%)$ \\
\hline $207(45.8 \%)$ \\
\hline $452(100 \%)$ \\
\hline $284(86.9 \%)$ \\
\hline $43(13.1 \%)$ \\
\hline $327(100 \%)$ \\
\hline $6(1.3 \%)$ \\
\hline $347(98.7 \%)$ \\
\hline $453(100 \%)$ \\
\hline
\end{tabular}

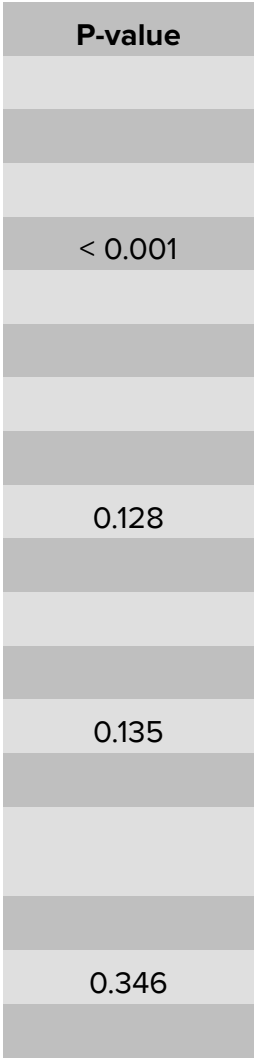

\section{Discussion}

This study sets out to describe the hematological changes in $P$. falciparum patients in Elnihoud Teaching Hospital, the study results revealed that there was a significant positive association between thrombocytopenia and $P$. falciparum malaria ( $P$-value 0.008$)$, which is consistent with $[7,13,14]$, who stated that the most common complications of falciparum malaria is thrombocytopenia and platelet dysfunction. The thrombocytopenia is usually not associated with bleeding even in patients with very severe form (platelets less $10 \times 10^{3} /$ microliter), and there was a complete recovery after treatment, so thrombocytopenia is a significant indicator for malaria, especially in correlation with typical fever and hypotension. Also, the study shows a significant positive association between leucopenia and $P$. falciparum malaria ( $P$-value 0.001$)$, which is in agreement with Ladhani et al. [15], who reported that leucopenia and thrombocytopenia occurred with $P$. falciparum malaria, but these were not associated with death. The leucopenia occurred most probably due to a bone marrow suppression, resulting from cytokines 
TABLE 2: Comparison of hematological parameters of the study groups.

\begin{tabular}{l} 
Characteristic \\
Hb \\
Normal \\
Anemia \\
Polycythemia \\
Total \\
TWBC \\
Normal \\
Leucopenia \\
Leukocytosis \\
Total \\
PLT \\
Normal \\
Thrombocytopenia \\
Thrombocytosis \\
Total \\
McV \\
Normal \\
Microcytic \\
Macrocytic \\
Total \\
Relative lymphocytosis \\
Yes \\
No \\
Total \\
\hline
\end{tabular}

\begin{tabular}{|c|c|c|c|}
\hline Patient & Control & Total & P-value \\
\hline $275(77.9 \%)$ & $85(85 \%)$ & $360(79.5 \%)$ & \\
\hline $77(21.8)$ & $15(15 \%)$ & $92(20.3 \%)$ & \\
\hline $1(0.3 \%)$ & $\mathrm{O}(0 \%)$ & $1(0.2 \%)$ & 0.278 \\
\hline $353(100 \%)$ & 100 (100\%) & 453(100\%) & \\
\hline 293(83.2\%) & 69(69\%) & $362(80.1 \%)$ & \\
\hline $22(6.3 \%)$ & $5(5 \%)$ & $27(6 \%)$ & $<0.001$ \\
\hline $37(10.5)$ & $26(26 \%)$ & $63(13.9 \%)$ & \\
\hline $352(100 \%)$ & 100(100\%) & $452(100 \%)$ & \\
\hline $267(75.6 \%)$ & 79(79\%) & $346(76.4 \%)$ & \\
\hline $75(21.3 \%)$ & $12(12 \%)$ & $87(19.2 \%)$ & \\
\hline $11(3.1 \%)$ & $9(9 \%)$ & $20(4.4 \%)$ & 0.008 \\
\hline 353 (100\%) & 100 (100\%) & 453 (100\%) & \\
\hline $210(59.5 \%)$ & $42(42 \%)$ & $252(55.6 \%)$ & \\
\hline $140(39.7 \%)$ & $57(57 \%)$ & 197(43.5\%) & 0.008 \\
\hline $3(0.8 \%)$ & $1(1 \%)$ & $4(0.9 \%)$ & \\
\hline 353 (100\%) & 100 (100\%) & 453 (100\%) & \\
\hline $51(19.1 \%)$ & $1(2.3 \%)$ & $52(16.8 \%)$ & 0.004 \\
\hline 216(80.9\%) & 42(97.7\%) & 258(83.2\%) & \\
\hline $267(100 \%)$ & 43 (100\%) & 310 (100\%) & \\
\hline
\end{tabular}

TABLE 3: Patients response to anti-malaria therapy.

Characteristic
Leucopenia
Leukocytosis
Total
Thrombocytopenia
Thrombocytosis
Total

\begin{tabular}{|c|}
$\begin{array}{c}\text { Patient before } \\
\text { the therapy }\end{array}$ \\
\hline $22(37.3 \%)$ \\
$37(62.7 \%)$ \\
$59(100 \%)$ \\
$75(87.2 \%)$ \\
$11(12.8 \%)$ \\
$86(100 \%)$ \\
\hline
\end{tabular}

\begin{tabular}{|c|}
\hline $\begin{array}{c}\text { Patient two } \\
\text { weeks after the } \\
\text { therapy }\end{array}$ \\
\hline $2(28.6 \%)$ \\
\hline $5(71.4 \%)$ \\
\hline $7(100 \%)$ \\
$7(87.5 \%)$ \\
\hline $1(12.5 \%)$ \\
\hline $8(100 \%)$ \\
\hline
\end{tabular}

\begin{tabular}{c}
$\begin{array}{c}\text { Normal values } \\
\text { two weeks after } \\
\text { the therapy }\end{array}$ \\
$20(38.5 \%)$ \\
$32(61.5 \%)$ \\
$52(100 \%)$ \\
$68(87.2 \%)$ \\
$10(12.8 \%)$ \\
$78(100 \%)$ \\
\hline
\end{tabular}

P-value

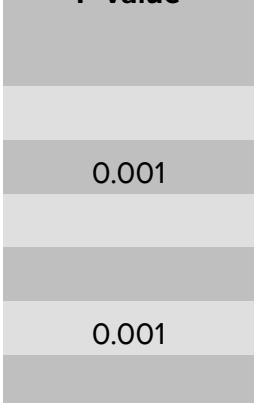

production and/or folate deficiency when associated with pancytopenia. There was a significant positive association between microcytic hypochromic red blood cell and $P$. falciparum malaria ( $P$-value 0.008 ) as it is stated by WHO [8] which reported the same point. Microcytic hypochromic red cells might due to either hepcidin production or loss of appetite and poor diet especially in childhood period. The study revealed 
a significant positive association between relative lymphocytosis and $P$. falciparum malaria ( $P$-value 0.004$)$, same result reported by Ladhani et al. [15]; this might be a part of the bone marrow failure which is associated with neutropenia because neutrophil has short half-life compared with lymphocytes and its deficiency appears early. There was no significant association with anemia and so pancytopenia because hemoglobin can take time to show a significant drop.

Two weeks after completing the treatment, patients with abnormal blood counts showed normal values that indicates that these patients should receive anti-malaria therapy irrespective of malaria-parasite results.

\section{Conclusion}

The study findings demonstrate that $P$. falciparum malaria is one of the common causes of hematological changes. The results showed that there was a statistically significant positive association between $P$. falciparum malaria and each of leucopenia, relative lymphocytosis, thrombocytopenia, and microcytic hypochromic red blood cells.

\section{Acknowledgement}

The authors would like to thank the members of the Department of Pathology, their colleagues, and the staff workers in medical laboratory of Elnihoud Teaching Hospital for their contribution and support.

\section{References}

[1] Kidist. M and Zerihun, Z. (2016). Thrombocytopenia as marker for the diagnosis of malaria among malaria suspected patients in Arba Minch Health Center, Gamo Gofa Zone, Southern Ethiopia: a cross-sectional study. African Journal of Science and Research, vol. 5, no. 1, pp. 61-64.

[2] Howes, R., Reiner, R., Battle, K., et al. (2015). Plasmodium vivax transmission in Africa. PLOS Neglected Tropical Diseases, vol. 9, no. 11, e0004222.

[3] WHO. (2018). World Malaria Report. National Malaria Programme.

[4] Bhupen, B., Prasanta, K., Krysh, G., et al. (2016). Acute pancreatitis in a patient with complicated falciparum malaria. Journal of Clinical and Diagnostic Research, vol. 10, no. 1 , pp. $18-20$. 
[5] Himanshu, A. and Seema, S. (2012). Thrombocytopenia in P. vivax malaria. NJIRM, vol. 2 , no. 3

[6] Jadhav, U., Patkar, V., and Kadam, N. (2004). Thrombocytopenia in malaria-correlation with severity and type of malaria. Journal of the Association of Physicians of India, vol. 52, pp. 615-618.

[7] Mast, Q., Groof, E., and Lenting, P. (2007). Thrombocytopenia and release of activated von Willebrand factor during early malaria. Journal of Infectious Diseases, vol. 196, pp. 622-628.

[8] WHO. (2010). Guidelines for the Treatment of Malaria. World Health Organization.

[9] Mast, Q., Groot, P., Heerde, W., et al. (2010) Thrombocytopenia in early malaria is associated with GP1b shedding in absence of systemic platelet activation and consumptive coagulopathy. British Journal of Haematology, vol. 151, pp. 495-503.

[10] Kreil, A., Wenisch, C., Brittenham, G., et al. (2000). Thrombopoietin in plasmodium falciparum malaria. British Journal of Haematology, vol. 109, pp. 534-536.

[11] Lowenberg, C., Charunwatthana, P., Cohen, S., et al. (2010). Severe malaria is associated with a deficiency of von Willebrand factor cleaving protease, ADAMTS13. Thrombosis and Haemostasis, vol. 103, pp. 181-187.

[12] Saravu, K., Rishikesh, K., and Kamath, A. (2014). Determinants of mortality, intensive care requirement and prolonged hospitalization in malaria - a tertiary care hospital based cohort study from South-Western India. Malaria Journal, vol. 13, no. 1, p. 370.

[13] Divya, R. and Sudha, R. (2016). A clinical study of falciparum malaria with special reference to its complications among malaria patients. Journal of Health Sciences, vol. 1, no. 4.

[14] Ladhani, S., Lowe, B., Cole, A. O., et al. (2002). Changes in white blood cells and platelets in children with falciparum malaria: relationship to disease outcome. British Journal of Haematology, vol. 119, no. 3, pp. 839-847. 